UDC 537.874; 621.396

\title{
VOLTAGE TUNABLE MICROWAVE MULTIBAND FILTERS THROUGH META-INTERFEROMETER
}

\author{
G. A. Kraftmakher, V. S. Butylkin, Y. N. Kazantsev, V. P. Mal'tsev \\ Fryazino Branch of Kotelnikov Institute of Radioengineering and Electronics of Russian \\ Academy of Sciences, Vvedensky Sq.1, Fryazino Moscow region 141190, Russia
}

The paper is received on September 15, 2018

\begin{abstract}
First it has been observed that microwave interferometer with embedded metastructure, meta-surface or meta-atom as voltage-controlled original beam-splitter can show new functionalities, such as specific selective control of line width and intensity, as well line shape and position in multiband spectral filtering. We realize and investigate modified meta-interferometer on basis of rectangular waveguide tee with varactor-loaded conductive resonance elements as a beam splitter at $3-6 \mathrm{GHz}$. It is achieved tunable considerable narrowing and $0.15-0.2 \mathrm{GHz}$ shifting of band; switching between stop-band $(-25 \mathrm{~dB})$ and pass-band $(-2 ;-5 \mathrm{~dB})$, as well widening and intensification of each filtering band by turns in dependence on resonance element properties and bias voltage $0-10 \mathrm{~V}$. The results open up a new approach to implement switchable and tunable multiband microwave filters.
\end{abstract}

Key words: metastructure, microwave, interferometer, beam splitter, tuning, varactor, multiband filters.

\section{Introduction}

At present microwave tunable multiband spectral filters, required in multifunctional wireless communication, are subjects of great interest [1]. They play important role for channel selection and signal processing. Basis of the microwave multiband filter is a cascade of different resonators in combination with transmission line. To select required filter electromechanical [2] or electrical switch by varactor diodes [3] is usually used. A simple compact single-band tunable metamaterials filter consists of a micro-strip transmission line with a varactor-loaded split ring resonator 
[4]. This approach has been developed and applied in [5-9]. In [5, 6] it has been achieved good band characteristics. In [7] it has been implemented dual-band tunable microwave filters based on two cascaded sections. In $[8,9]$ it is proposed a metastructure making it possible to achieve nonreciprocal microwave filter that can be controlled by an electrostatic field. But it is very difficult to achieve both tunable and multiband spectral filtering [10].

In this work we implement tunable microwave multiband filtering through modified interferometer on basis of rectangular waveguide tee in which metastructure is embedded as a tunable beam splitter; we will call "meta-interferometer". Concept of tunable beam splitter based on electro-optical materials embedded in optical Mach-Zehnder interferometer is in progress in classical photonics for quantum information processing [11]. Here we present measurement results of electromagnetic waves transmission coefficient $T$ in meta-interferometer with different beam-splitters at $3-6 \mathrm{GHz}$. In the first variant varactor-loaded dipole "Butterfly (excited by microwave electric field $E$ ) is used (Fig. $1 a, b$ ). In the second variant beam splitter is a ray of varactor-loaded twice split rings, excited by microwave magnetic field $h$ (Fig. 1c).

\section{Proposed meta-interferometer}

Empty rectangular waveguide $h$-plane tee (Port 1 is input) is transformed into interferometer and acts as multiband filter in frequency dependence of transmission in the cases when Port 2 (or 3 ) is short circuited. In order to increase a number of interference bands it is necessary to increase a length of short circuited Arm. Metainterferometer (Fig. $1 a$-scheme, $b, c$-photographs) contains addition: metastructure as a tunable beam-splitter.

That is individual conductive resonant element in the form of varactor-loaded dipole "Butterfly" or array of twice split rings with varactors. Short circuited side $h$ Arm (24x48 mm) has length $250 \mathrm{~mm}$ and is removable; Port 2 is output.

For tuning we use varactor MA46H120 (MACOM), with capacitance varying from 1 to $0.15 \mathrm{pF}$ by supplying back bias voltage $V_{\mathrm{DC}}$ from 0 to $10 \mathrm{~V}$. Varactor is welded into the gap of dipole or split ring. We investigate also three dipoles with 
fixed capacitance $C_{1}=6.8 \mathrm{pF} ; C_{2}=1$; and $C_{3}=0.1 \mathrm{pF}$ ( $C$-loaded "Butterfly"). Two variants of meta- interferometer, differing in type of beam splitter, are investigated.

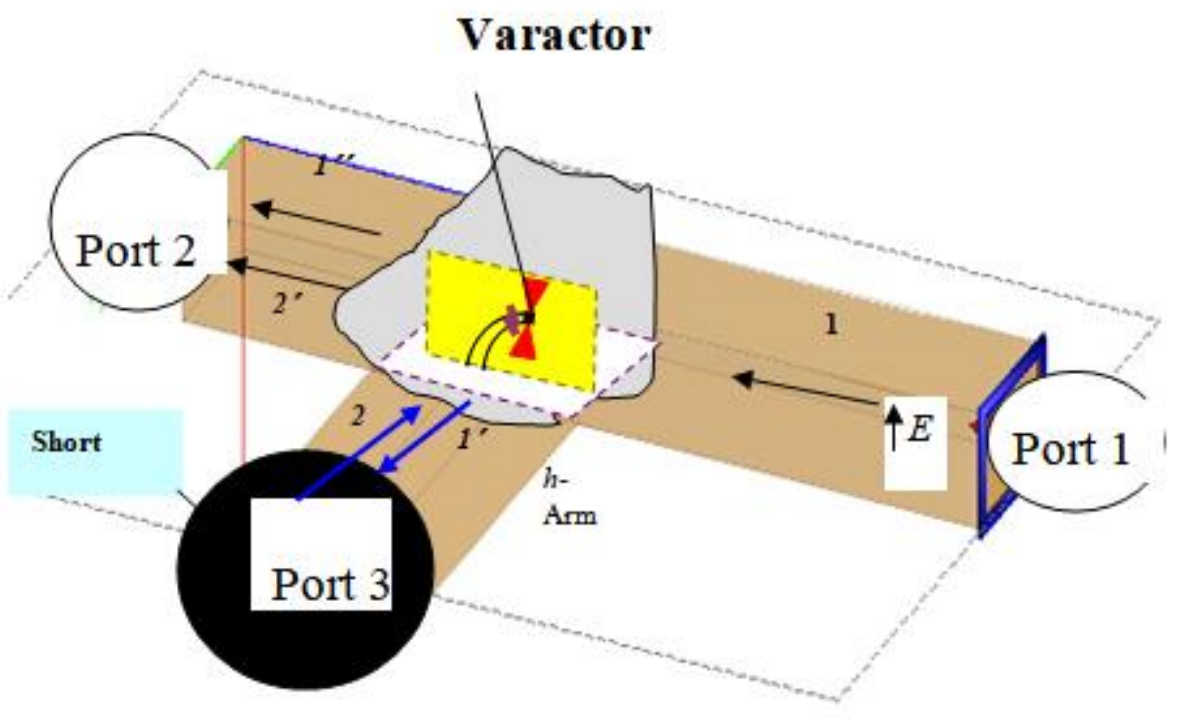

$a$

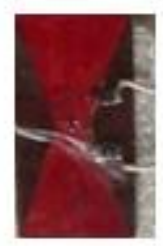

$b$

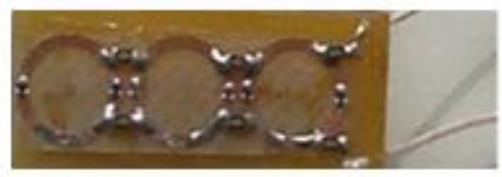

C

Fig. 1. Proposed meta-interferometer:

$a$ with dipole "Butterfly" as a beam splitter (scheme)

$b$ dipole "Butterfly" (photo)

$c$ a ray of magnetically excited twice split rings (photo)

In the first variant copper $C$-loaded or varactor-loaded dipole "Butterfly" (20x10 $\mathrm{mm}$ ) on $2 \mathrm{~mm}$-thick textolite substrate is used. In the second variant beam splitter is a ray of copper twice split rings TSR (diameter of $6.6 \mathrm{~mm}$, each ring contains two varactors). The sizes of elements are chosen so that the resonance response of transmission coefficient $T$ will be observed at frequency in the given range $3-6 \mathrm{GHz}$ of the voltage standing wave ratio (VSWR) panoramic measurer. Resonance element is placed along axis of main waveguide 1 across the side $h$-Arm. Meta-interferometer acquires new functionalities due to resonance properties of beam-splitter and superposition of transmitted, reflected and re-reflected waves. In this case periodicity 
violation, change of shape and position of interference bands and possibility of multifarious control is observed in dependence on resonance properties of beamsplitter (width, intensity and frequency of the resonance)

\section{Measurements results}

With dipole "Butterfly" as a beam splitter: Figs. $2 a$, $b$ show typical resonance dependencies of $T$ (dipole resonances DR) in rectangular waveguide (WG) with different dipoles "Butterfly": $C$-loaded (Fig. 2a) and varactor-loaded (Fig. 2b).

The DR can be tuned by the use of different $C$-dipoles with different fixed capacitances or with varactor-loaded "Butterfly" by the application of a bias voltage $V_{\mathrm{DC}}$ to varactor. Meta-interferometer shows multiband spectral filtering, different from five-band spectrum of empty interferometer (EI). In meta-interferometer passbands narrowing and stop-bands (F) widening is observed (Fig. 2c,e). ). Besides, meta- interferometer acquires new capability, such as selective specific control covering several interference bands because of influence of wide dipole resonance DR. In this case intensity, width and frequency $\left(f_{\mathrm{i}}\right)$ of different interference bands are controlled differently in dependence on DR position $\left(f_{\mathrm{DR}}\right)$. So, Figs. $2 c, d$ show that in the case of $C$-loaded "Butterfly" with decrease of $C$ from $6.8 \mathrm{pF}$ to $1 \mathrm{pF}$ it is observed intensity decrease and narrowing of stop-band $F_{1}$ and simultaneously widening of bands $\mathrm{F}_{3}$ and $\mathrm{F}_{4}$; at the same time $\mathrm{F}_{1}$ shifts more than other bands, while $\mathrm{F}_{5}$ position is practically not changed. With $C=0.1 \mathrm{pF}$ it is observed level increase and widening of pass - bands, as well stop-bands narrowing and $\mathrm{F}_{5}$ disappearance.

Switch between stop $(-30 \mathrm{~dB})$ and pass-bands $(-2 ;-5 \mathrm{~dB})$ takes place with change $C$ from $1 \mathrm{pF}$ to $0.1 \mathrm{pF}$. Similar specific selective and switchable controllability is observed in the case of varactor-loaded "Butterfly" (Fig. $2 e, f$ ). Pass-bands narrowing and stop-bands widening about EI are different for different interference bands (Fig. $2 e$ ). By variation of $V_{\mathrm{DC}}$ from $0 \mathrm{~V}$ to $10 \mathrm{~V}$ increase of level and widening of pass-bands as well considerable narrowing and shifting of stopbands occurs (Fig. 2 f); at the same time different bands are tuned differently. Really, band $F_{1}(3.66 \mathrm{GHz})$ is not shifting, while bands $F_{2}(3.96 \mathrm{GHz}) ; F_{3}(4.38 \mathrm{GHz})$.and $F_{4}$ $(4,78 \mathrm{GHz})$ shift to $4.14 ; 4.6$ and $5.1 \mathrm{GHz}$ on $0.18 ; 0.2$ and $0.3 \mathrm{GHz}$ and switch 
between stop- $(-20 ;-25 \mathrm{~dB})$ and pass-bands $(-5 \mathrm{~dB})$ takes place.
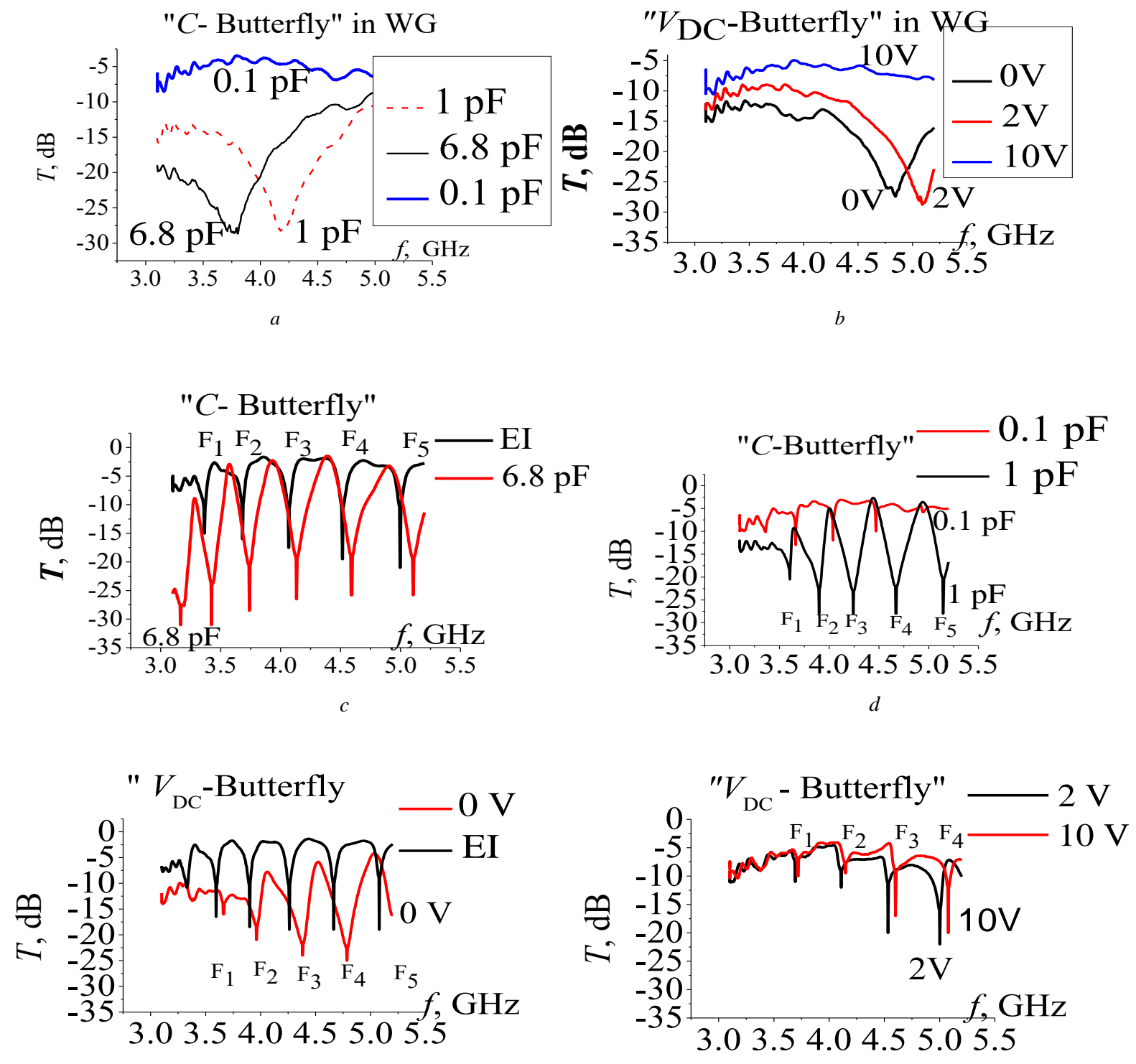

Fig. 2. Dipole "Butterfly": Measured transmission T $a \quad \mathrm{WG}, C_{1}=6.8 \mathrm{pF} ; C_{2}=1 \mathrm{pF} ; C_{3}=0.1 \mathrm{pF}$ $b \quad \mathrm{WG}, V_{\mathrm{DC}}=0 ; 2 ; 10 \mathrm{~V}$

$c$ Meta-interferometer. $C_{1}=6.8 \mathrm{pF}$ in comparison with EI $d$ Meta-interferometer. $C_{2}=1 ; C_{3}=0.1 \mathrm{pF}$

e Meta-interferometer. $V_{\mathrm{DC}}=0 \mathrm{~V}$ in comparison with EI $f$ Meta-interferometer. $V_{\mathrm{DC}}=0 ; 2 ; 10 \mathrm{~V}$ 


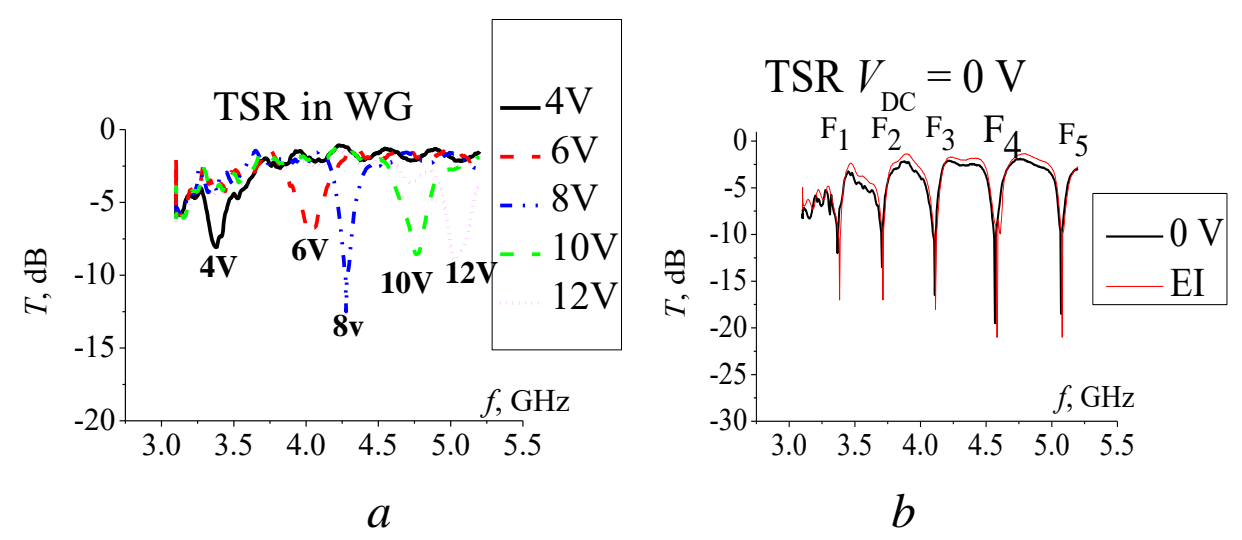

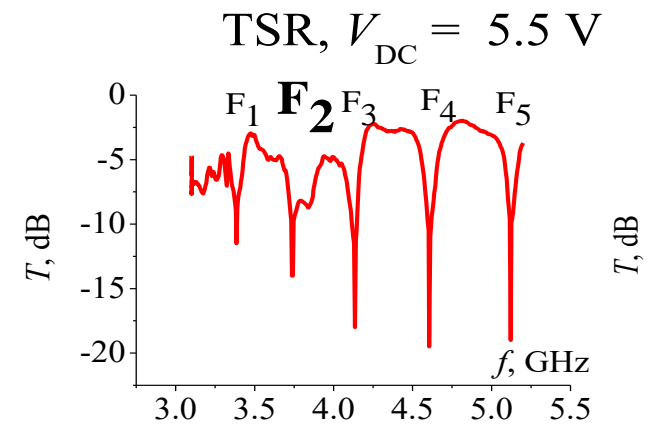

$c$

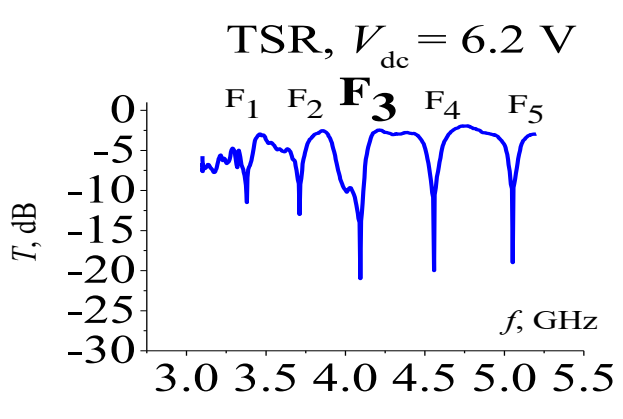

$d$

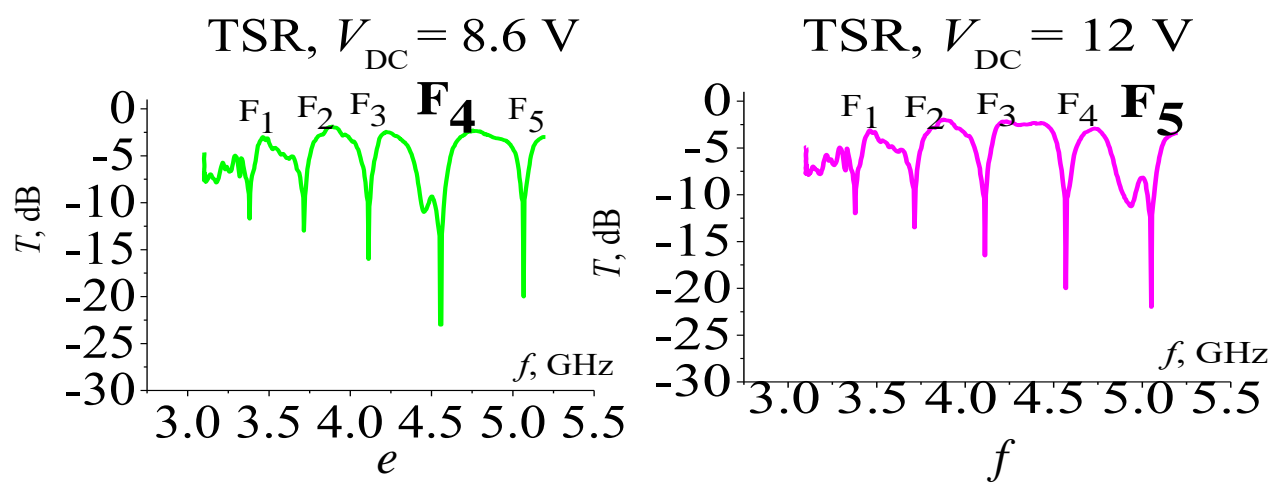

Fig. 3. Twice split rings TSR. Measured transmission $T$ $a \mathrm{WG}, V_{\mathrm{DC}}=4 ; 6 ; 8 ; 10 ; 12 \mathrm{~V}$

$b$ Meta-interferometer. $V_{\mathrm{DC}}=0 \mathrm{~V}$ in comparison with EI

$c$ Meta-interferometer. $V_{\mathrm{DC}}=5.5 \mathrm{~V}$; change of $\mathrm{F}_{2}$

$d$ Meta-interferometer. $V_{\mathrm{DC}}=6.2 \mathrm{~V}$; change of $\mathrm{F}_{3}$

e Meta-interferometer. $V_{\mathrm{DC}}=8.6 \mathrm{~V}$; change of $\mathrm{F}_{4}$

$f$ Meta-interferometer. $V_{\mathrm{DC}}=12 \mathrm{~V}$; change of $\mathrm{F}_{5}$

With twice split rings TSR: Results of measurement of frequency dependences of $T$ in WG with array of varactor-loaded TSR show magnetic resonance (MR) due to ring current induced by $h$-field. The MR shifts to higher frequencies with increase of 
$V_{\text {DC }}$ (Fig. $3 a$ ). In Fig. $3 b$ we see that when TSR are embedded in EI, interference dependence of $T$ is not practically changed. With $V_{\mathrm{DC}}$ variation moving MR can approach individual interference band position. In this case corresponding band is controlled separately without changes of other bands. One can achieve widening and intensification of each stop-band by turns. Fig. $3 c$ demonstrates change of $\mathrm{F}_{2}$ at $V_{\mathrm{DC}}$ $=5.5 \mathrm{~V}$. In Fig. $3 d$ one can see change of $\mathrm{F}_{3}$ at $V_{\mathrm{DC}}=6.2 \mathrm{~V}$. It has been observed also that changes of $\mathrm{F}_{4}$ and $\mathrm{F}_{5}$ occur at $V_{\mathrm{DC}}=8.6 \mathrm{~V}$ and $12 \mathrm{~V}$.

\section{Conclusion}

So, meta-interferometer on basis of rectangular waveguide tee with varactorloaded dipole "Butterfly" or a ray of twice split rings as an original tunable beam splitter shows specific selective electric controllability of multiband spectral filtering at $3-6 \mathrm{GHz}$ in dependence on resonance properties of beam splitter and voltage variation from 0 to $10 \mathrm{~V}$ : selective tuning covering several stop-bands; tunable considerable narrowing and $0.15-0.2 \mathrm{GHz}$ shifting of defined stop-band; switch between stop (-20; $-25 \mathrm{~dB})$ and pass-bands $(-5 \mathrm{~dB})$, with "Butterfly"; as well selective controlling of individual band by TSR. The obtained results open up new approach to design microwave multifarious multiband spectral filtering and implement selective voltage tuning, required in multifunctional and multichannel wireless communication.

\section{References}

1. Cameron Richard J., Kudsia Chandra M., and Mansour Raafat R. Microwave Filters for Communication Systems: Fundamentals, Design, and Applications, Second Edition, JohnWiley \& Sons, 2018

2. Entesari K. and Rebeiz G.M.: 'A differential 4-bit 6.5-10-GHz RF MEMS tunable filter', IEEE Trans. Microwave Theory Tech., 2005, Vol. 53, No. 5, pp. 1103 -1110, doi: $\quad$ 10.1109/TMTT.2005.843501

3. Brown A.R., and Rebeiz G.M.: 'A varactor-tuned RF filter', IEEE Trans Microwave Theory Tech, 2000, Vol. 48, No. 7, pp. 1157-1160, doi: $\underline{10.1109 / 22.848501}$ 
4. Gil I., Garcia-Garcia J., Bonache J., Martin F., et al.: 'Varactor-loaded split ring resonators for tunable notch filters at microwave frequencies', Electronics Letters, 2004, Vol. 40, No. (21, pp.1347 -1348, doi: 10.1049/el:20046389

5. Genc Alper, and Baktur Reyhan: 'A tunable bandpass filter based on varactor loaded split-ring resonators', Microwave and optical technology letters, 2009, Vol. 51, No. 10, pp. 2394 -2396, doi: 10.1002/mop.24641

6. Zhao Ya-juan, Zhou Bi-cheng, Zhang Ze-kui I, et al.: 'A compact tunable metamaterial filter based on split-ring resonators', Optoelectronics letters, 2017, Vol. 13, No. (2, pp. 120 -122, doi: 10.1007/s11801-017-7008-7

7. Vendik I., Kholodnyak D., Kapitanova P., et al.: 'Multiband tunable microwave resonators and filters on a combination of right/left-handed transmission line sections'. Proceedings of $3^{\text {rd }}$ International Congress on Advanced Electromagnetic Materials in Microwaves and Optics, London, 2009, pp. 181-183

8. Kraftmakher G.A., Butylkin V.S., and Kazantsev Yu.N.: 'Electrically controlled frequency bands of nonreciprocal passage of microwaves in metastructures', Tech. Phys. Letters, 2013, Vol. 39, No. 6, pp. 505-508, doi: 10.1134/S1063785013060060 9. Butylkin, V.S., G.A. Kraftmakher and Yu.N. Kazantsev, “About electric control of nonreciprocity of microwave transition in metastructures," Zhurnal radioelektroniki Journal of Radio Electronics, 2014, No. 12. Available at http://jre.cplire.ru/jre/dec14/22/text.html (In Russian)

10. Fok Mable P. and Ge Jia: 'Tunable Multiband Microwave Photonic Filters', Photonics, 2017, Vol. 4, No. 4, p. 45, doi: 10.3390/photonics4040045

11. Ma Xiao-song, Zotter Stefan, Tetik Nuray, et al.: 'A high-speed tunable beam splitter for feed-forward photonic quantum information processing', Optics express, 2011, Vol. 19, No. 23, pp. 22723 -22730, doi: $\underline{10.1364 / O E .19 .022723}$

\section{For citation:}

G. A. Kraftmakher, V. S. Butylkin, Y. N. Kazantsev, V. P. Mal'tsev. Voltage tunable microwave multiband filters through meta-interferometer. Zhurnal Radioelektroniki - Journal of Radio Electronics. 2018. No. 9. Available at http://jre.cplire.ru/jre/sep18/19/text.pdf

DOI 10.30898/1684-1719.2018.9.19 